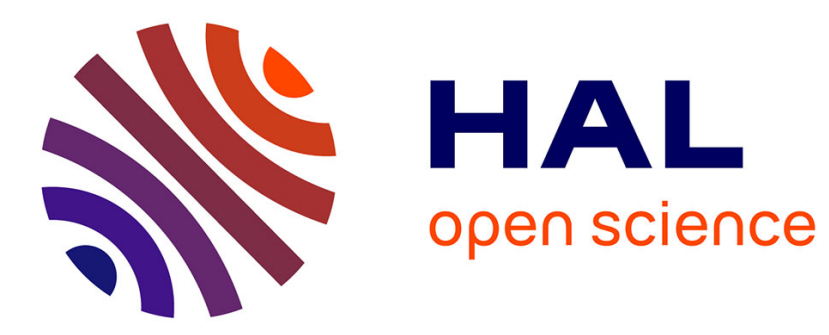

\title{
Comparative Analysis of Median and Average Filters in Impulse Noise Suppression
}

\author{
Luyao Shi, Wenlong Yuan, Libo Zhang, Benqiang Yang, Yang Chen, \\ Huazhong Shu, Limin Luo, Jean-Louis Coatrieux
}

\section{To cite this version:}

Luyao Shi, Wenlong Yuan, Libo Zhang, Benqiang Yang, Yang Chen, et al.. Comparative Analysis of Median and Average Filters in Impulse Noise Suppression. Fluctuation and Noise Letters, 2015, 14 (01), pp.1550002. 10.1142/S0219477515500029 . hal-01113271

\section{HAL Id: hal-01113271 \\ https://hal.science/hal-01113271}

Submitted on 9 Mar 2015

HAL is a multi-disciplinary open access archive for the deposit and dissemination of scientific research documents, whether they are published or not. The documents may come from teaching and research institutions in France or abroad, or from public or private research centers.
L'archive ouverte pluridisciplinaire HAL, est destinée au dépôt et à la diffusion de documents scientifiques de niveau recherche, publiés ou non, émanant des établissements d'enseignement et de recherche français ou étrangers, des laboratoires publics ou privés. 


\title{
Comparative Analysis of Median And Average Filters in Impulse Noise Suppression
}

\author{
Luyao Shi ${ }^{1,2,3}$, Wenlong Yuan ${ }^{1,2,3}$, Libo Zhang ${ }^{4}$, BenQiang Yang ${ }^{4}$,Yang Chen ${ }^{1,2,3}$, \\ Huazhong Shu ${ }^{1,2,3}$, Limin Luo ${ }^{1,2,3}$, Jean-Louis Coatrieux ${ }^{3.5,6}$
}

1. Laboratory of Image Science and Technology, Southeast University, Nanjing, China;

2. the Key Laboratory of Computer Network and Information Integration (Southeast University), Ministry of Education, China

3. Centre de Recherche en Information Biomedicale Sino-Francais (LIA CRIBs), Rennes, France;

4. Department of radiology, General Hospital of Shenyang Military Area Command, Shenhe District Shenyang, China.

5. INSERM, U1099, Rennes, F-35000, France;

6. Université de Rennes 1, LTSI, Rennes, F-35000, France.

Abstract-Median type filters take the main stream in suppressing impulse noise, and the Laplacian distribution assumption lays the basis for it. We however demonstrate in this paper that the Gaussian distribution assumption is more preferable than Laplacian distribution assumption in suppressing impulse noise, especially for high noise densities. This conclusion is supported by numerical experiments with different noise densities and filter models

Index Terms-Median filter, Impulse noise, Salt and pepper noise, Gaussian distribution, Laplacian distribution.

\section{INTRODUCTION}

Median type filters are well known as the effective methods to suppress impulse noise which often arises from sensor damage, malfunctioning or timing errors in signal acquisition [1]. Suppressing impulse noise routinely includes two stages - noise detection and noise removal [2-4]. The Standard Median Filter (SMF) is ineffective in the cases with high noise densities, and often severely suffers from detail loss and artifacts [5]. Adaptive Median Filter (AMF) was proposed in [6] to improve the performance of median filter by only replacing corrupted pixel values by median values while leaving uncorrupted pixels unchanged. Additionally, the AMF recursively enlarges the filter window until one uncorrupted median is identified. The Decision Based Algorithm (DBA) proposed in [7] is similar to the AMF method except that DBA replaces the corrupted pixel value by a neighborhood pixel value when the median is tagged as a corrupted pixel. In [8], a method named Modified Decision Based Unsymmetric Trimmed Median

This research was supported by National Basic Research Program of China under grant (2010CB732503), National Natural Science Foundation under grants (81000636, 81370040), and the Project supported by Natural Science Foundations of Jiangsu Province (BK2011593). 
Filter (MDBUTMF) was developed to improve DBA method by removing uncorrupted pixels in median calculation and setting the corrupted pixel value to mean of the selected window when all the window elements are corrupted. To achieve edge-preserving noise suppression, Chan and Nikolova put forward in [9] a two-phase algorithm which includes a SMF based noise detection and an edge-preserving term regularized optimization. In [10], a multi-scale adaptive median-based filtering was developed to suppress salt-and-pepper noise.

Laplacian distribution is the basis assumption for the median operation in median type filters [11]. But in fact, the exact distribution for the 2D neighboring intensities is always unknown. Large variance or error will be produced if unsuitable distribution assumption is chosen. In this paper we would argue that Gaussian distribution assumption (for average filter) theoretically allows a better estimation of corrupted points than the Laplacian distribution assumption (for median filter). A thorough analysis based on simple median and average filters is presented in both 1D and 2D domain. This paper is organized as follows: in section II, we analyze and compare the MLE using Gaussian and Laplacian distributions under different noise levels. Comparative experimental results are given in section III. Section IV concludes this paper with a brief description of its contributions and some open questions for future work.

\section{LAPLACIAN OR GAUSSIAN DISTRIBUTION}

Based on the theory of statistical estimation, the sample median corresponds to the maximum likelihood estimators of locations for independent and identically distributed (i.i.d.) observations obeying the Laplacian distributions with probability density function (pdf) shown in Fig.1 [11]. Let $x_{1}, \ldots ., x_{N}$ be $N$ i.i.d. Laplacian distributed observations with unknown location parameter $\mu$ and the same variance $2 \eta^{2}$, the MLE of $\mu$ is denoted by $\hat{\mu}$, which maximizes the likelihood function:

$$
\begin{aligned}
L\left(x_{1}, \ldots, x_{N} ; \mu\right) & =\prod_{i=1}^{N} f\left(x_{i}-\mu\right) \\
& =\left(\frac{1}{2 \eta}\right)^{N} \exp \left(-\sum_{i=1}^{N}\left|x_{i}-\mu\right| / \eta\right)
\end{aligned}
$$

Maximizing the likelihood function (1) is equivalent to minimizing (2):

$$
\sum_{i=1}^{N}\left|x_{i}-\mu\right| / 2 \sigma^{2}
$$

Minimizing (2) with respect to $\mu$ leads to the simple median $\hat{\mu}=\operatorname{MEDIAN}\left(x_{1}, \ldots ., x_{N}\right)[12]$. Here for an ordered list, the MEDIAN operator is defined as producing the middle value for odd value numbers or the arithmetic mean of the middle two observations for even value numbers. Assuming the Laplacian distribution of the observations in the $3 \times 3$ window, the $2 \mathrm{D}$ median 
filtering of the center pixel value $\mu$ is in fact the maximizer of the likelihood function with respect to each location parameter $\mu[13]$.

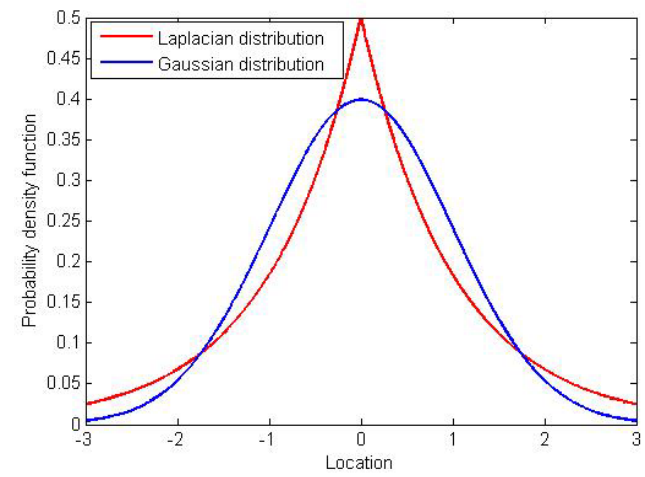

Fig. 1. The probability density functions for Laplacian and Gaussian distributions.

Likewise, assuming the Gaussian distribution shown in Fig. 1, we can build the likelihood function in (3).

$$
\begin{aligned}
L\left(x_{1}, \ldots, x_{N} ; \mu\right) & =\prod_{i=1}^{N} f\left(x_{i}-\mu\right) \\
& =\left(\frac{1}{2 \pi \sigma^{2}}\right)^{N / 2} \exp \left(-\sum_{i=1}^{N}\left(x_{i}-\mu\right)^{2} / 2 \sigma^{2}\right)
\end{aligned}
$$

The value $\mu$ minimizing (3) leads to the average operator:

$$
\bar{\mu}=\sum_{i=1}^{N} x_{i} / N
$$

Then we can easily obtain the variance $\sigma_{\bar{\mu}}^{2}$ of $\bar{\mu}$ :

$$
\sigma_{\bar{\mu}}^{2}=\sigma^{2} / N
$$

Here, equation (5) shows that the variance estimation for Gaussian distribution decreases linearly with the available observation numbers.

It is however nontrivial to estimate the variance of the median $\hat{\mu}$ as the MLE for Laplacian distributions. In [14], an equation shown in (6) was derived to approximate the variance $\sigma_{\hat{\mu}}^{2}$ of median $\hat{\mu}=\operatorname{MEDIAN}\left(x_{1}, \ldots, x_{N}\right)$ :

$$
\sigma_{\hat{\mu}}^{2} \approx \eta^{2} /(N-0.5)
$$

where $2 \eta^{2}$ is the variance of Laplacian distributed observations $x_{1}, \ldots ., x_{N}$. And in [16], Lee and Tantaratana proposed a more precise variance approximation for median: 


$$
\sigma_{\hat{\mu}}^{2}\left\{\begin{array}{cc}
=2 \eta^{2} & N=1 \\
\approx 0.64 \eta^{2} & N=3 \\
\approx 4 \eta^{2} / 3(N-5 / 4) & N=5,7,9 \ldots .
\end{array}\right.
$$

\section{Simulated EXPERIMENT}

\section{A. 1D Simulation}

Median and average operators respectively lead to the MLE $\hat{\mu}$ and $\bar{\mu}$ of location parameter $\mu$ for Gaussian and Laplacian distributions. Equations (5) and (7) unveil that the variances for the $\hat{\mu}$ and $\bar{\mu}$ are in reciprocal ratio of the observation number $N$. Fig. 2 (a) plots the variances with respect to the observation number $N$ when applying median and average operators to estimate the true $\mu$ for the simulated Gaussian and Laplacian distributed random values. We set the true $\mu$ and variance to 0 and 1 to give typical simulations of Gaussian and Laplacian distributed random values, and $1 \times 10^{7}$ points are generated for each observation to alleviate the randomness in simulation. The variance $\sigma^{2}$ of the MLE for the simulation is calculated via (8):

$$
\sigma^{2}=\frac{1}{M} \sum_{j=1}^{M}\left(\theta_{j}-\bar{\theta}\right)^{2}
$$

where $M$ denotes the total $1 \times 10^{7}$ simulations for each observation, and $\theta$ corresponds to the estimation $\hat{\mu}$ or $\bar{\mu}$ which are obtained by applying median or average operators on the $N$ observations in each of the $1 \times 10^{7}$ simulations. $\bar{\theta}$ is the mean of $\theta$ for all the $1 \times 10^{7}$ simulation, and can be considered a good estimation of the true location parameter ( $\bar{\theta}$ approaches the true value if the simulation number increases to infinity). For even observation numbers, we calculate the median $\hat{\mu}$ as the arithmetical mean of the two middle-valued data. Fig. 2 (a) shows that, for both median and average operators, estimation variances drop when more observations are available. We also note in Fig. 2 (a) that median operator on the matched Laplacian simulation leads to a little lower variance than average operator on the matched Gaussian simulation.

For practical situation the distribution assumptions should be chosen based on the properties of the target data. Using $2 \mathrm{D}$ impulse noise suppression as an example, the average filtering is applicable for regions of smooth intensity variation (e.g. backgrounds), and the median filtering is suitable for restoring regions with rapid intensity variation (e.g. edges). Unfortunately, we often have no prior distribution knowledge of the data to be estimated. Large variance or error will be produced if unsuitable distribution is assumed. 

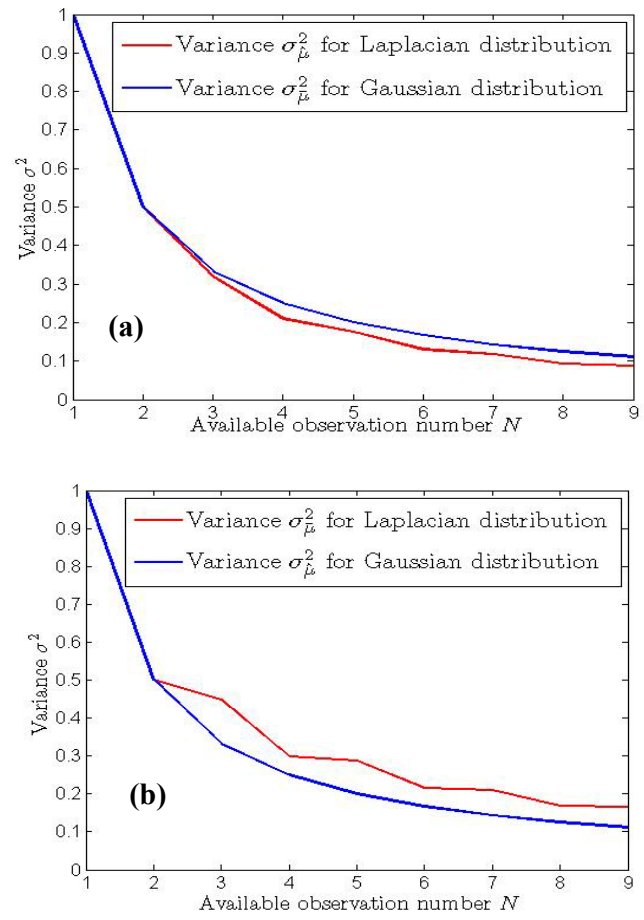

Fig. 2. The calculated variances ( $\sigma_{\hat{\mu}}^{2}$ and $\sigma_{\bar{\mu}}^{2}$ ) of $\hat{\mu}$ and $\bar{\mu}$ with respect to observation number $N$ for the following two cases. (a) and (b) correspond to matched and unmatched operations, respectively: (a) applying median operator to Laplacian distributed random values (red), and applying average operator to Gaussian distributed random values (blue). (b) applying average operator to Laplacian distributed random values (blue), and applying median operator to Gaussian distributed random values (red).

Fig. 2 (b) illustrates the calculated variances $\sigma_{\bar{\mu}}^{2}$ and $\sigma_{\hat{\mu}}^{2}$ when respectively applying average and median operators on simulated Laplacian and Gaussian distributed random values. Referring to the result in Fig.2 (a), we can note in Fig.2 (b) a significant variance increase when applying median operator to the unmatched Gaussian distributed data. On the contrary, the variance stays unchanged if we apply average operator to the unmatched Laplacian distributed data.

\section{B. 2D Simulation}

From above we know that, with unmatched distribution assumptions, larger variance can be induced by median operator than average operator. Median based filtering has long been considered the first choice in estimating unknown pixels contaminated by impulse noise. Now a natural wondering is whether average operator can also outperform median operator in estimating 2D pixels. We replace the above 1D median and average operators by 2D median and average filters, and the above 1D vectors $\mu$ and $x$ are changed to neighboring pixels in 2D domain. The above $N$ observations are then transformed to 2D neighboring pixels within filtering window. For brevity, this 2D filtering window is also denoted by $N$, and set to $3 \times 3$ size in this simulation. We chose four $512 \times 512$ grayscale images (Lena, Plane, Peppers and Magnetic resonance imaging (MRI) brain images in Fig.3 (a)-(d)). The original intensity intervals of Lena, Plane and Peppers and the MRI images are $(0,255)$. We added salt-and-pepper noise with 
density varying from $0 \%$ to $90 \%$ (10\% increments) to simulate different observation numbers. Pixels corrupted by salt-and-pepper noise have pre-known intensities 255 or 0 . Only pixels with intensities other than 255 or 0 in the filter windows are considered uncorrupted and involved in estimation. Pixel values are kept unchanged if the current pixels are found uncorrupted or all the pixels are corrupted in filter window. We evaluate the estimation by MSE (mean squared error):

$$
\operatorname{MSE}=\frac{1}{|M|} \sum_{j=1}^{|M|}\left(\mu_{j}-I_{j}\right)^{2}
$$

where, $\mu_{j}$ and $I_{j}$ denote the pixel values in the restored image and the original clean image. $|M|$ is the total pixel number.

Fig. 4 (a)-(d) plot the calculated MSE with respect to noise densities when applying median and average filters to estimate corrupted pixels. We can see in Fig. 4 that as noise density increases (noise density increases from $10 \%$ to $90 \%$ ) the average filtering leads to restorations with significantly lower MSE than median filtering for all the four test images. This confirms the result in Fig. 2 again that median operator tends to produce large estimation error when the inherent intensity distribution is unknown. Restoration performances are quantitatively measured by the peak signal-to-noise ratio (PSNR) and the structural similarity index comparisons (SSIM) in [15]:

$$
\begin{gathered}
\operatorname{PSNR}(\mu, I)=10 \log _{10}\left(|M| \cdot 255^{2} / \sum_{j=1}^{|M|}\left(\mu_{j}-I_{j}\right)^{2}\right) \\
\operatorname{SSIM}(\mu, I)=\frac{\left(2 m_{\mu} m_{I}\right)\left(2 \sigma_{\mu I}+C_{2}\right)}{\left(m_{\mu}^{2}+m_{I}^{2}+C_{1}\right)\left(\sigma_{\mu}^{2}+\sigma_{I}^{2}+C_{2}\right)}
\end{gathered}
$$

where, $\mu$ and $I$ denote the restored image and the original true image. $m_{\mu}$ and $m_{I}$ denote the mean intensities of images $\mu$ and $I, \sigma_{\mu}$ and $\sigma_{I}$ are the standard deviation of images $\mu$ and $I, \sigma_{\mu I}$ is the covariance of images $\mu$ and $I, C_{1}=\left(K_{1} L\right)^{2}$ and $C_{2}=\left(K_{2} L\right)^{2}$ with $L$ the dynamic range of the pixel values (255 for 8-bit grayscale images), $K_{1}$ and $K_{2}$ are set to 0.01 and 0.03 as suggested in [15].

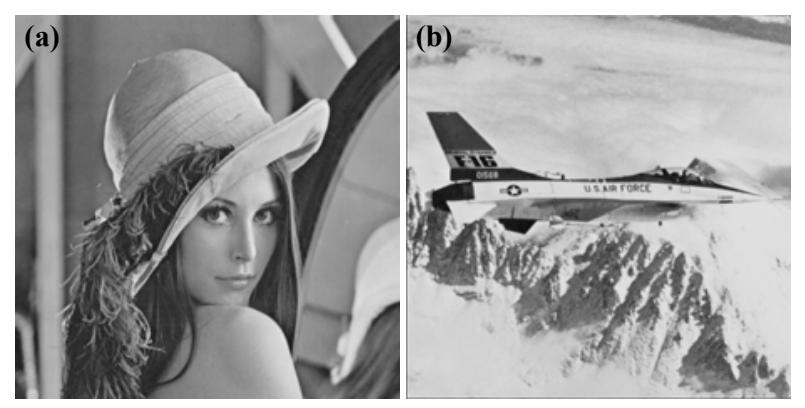




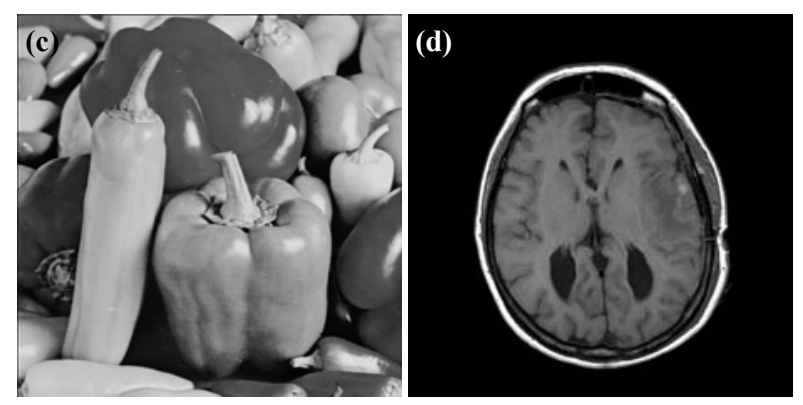

Fig. 3. Test images (a) Lena (b) Plane (c) Peppers and (d) MRI images.
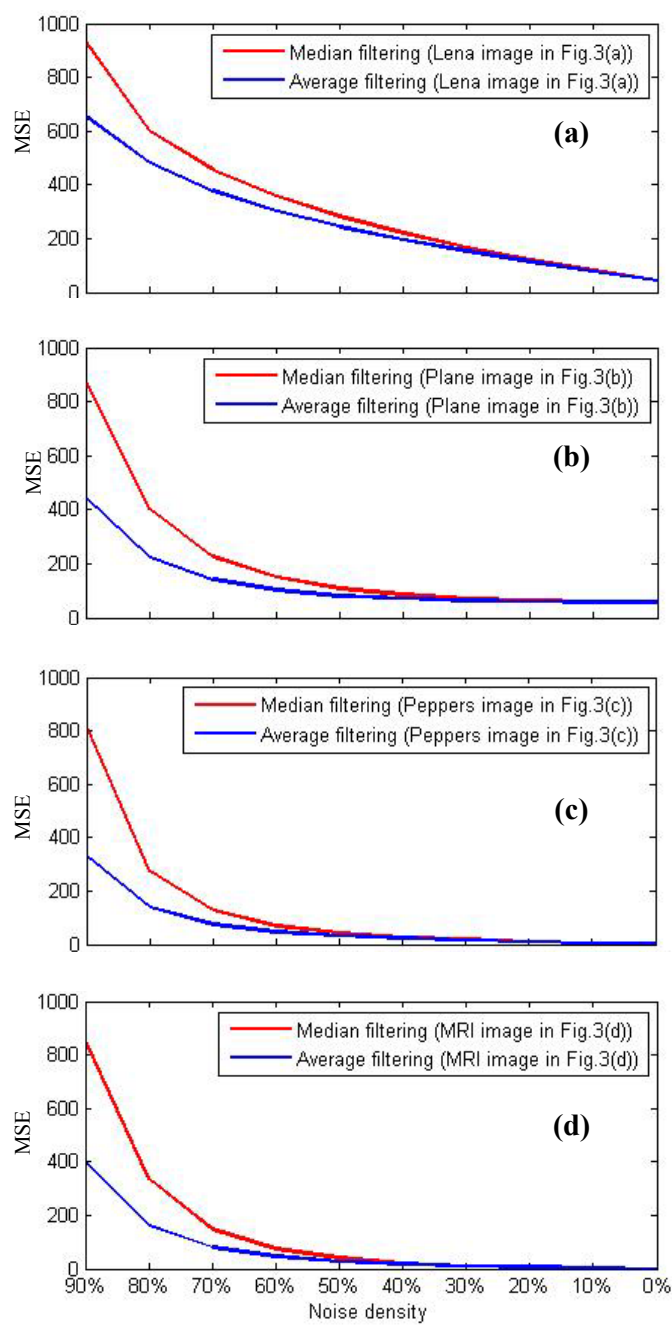

Fig. 4. The MSE versus noise densities when applying median and average filters to estimate the corrupted pixels in the four test images in Fig.3.

Fig. 5 and Fig. 6 illustrate the restoration results of Lena and MRI images with $10 \%$ to $90 \%$ noise densities for the above experiments with median and average filters. Zoomed image parts are given, and the calculated PSNR and SSIM values with respect to the original images are listed in the below captions. Fig. 5 (a) and Fig. 6 (a) are the original image parts for reference. We can find that average filter works better than the median filter method for almost all the noise densities, and this advantage becomes bigger as noise density increases (over $2.5 \mathrm{~dB}$ PSNR increasement for $90 \%$ noise density). The median filter only provides slightly 
higher PSNR values than average filter for 30\% noise density. The results in Fig.5 and Fig.6 are found consistent with the results in

Fig.4. Significantly better structure restoration and less introduced artifacts can be seen in the average filter results than the median filter results for high noise densities (see the arrows).

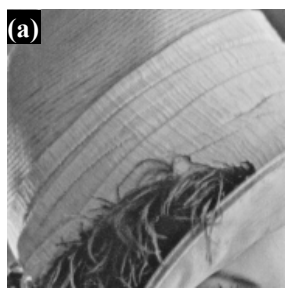

\section{Median filter Average filter}
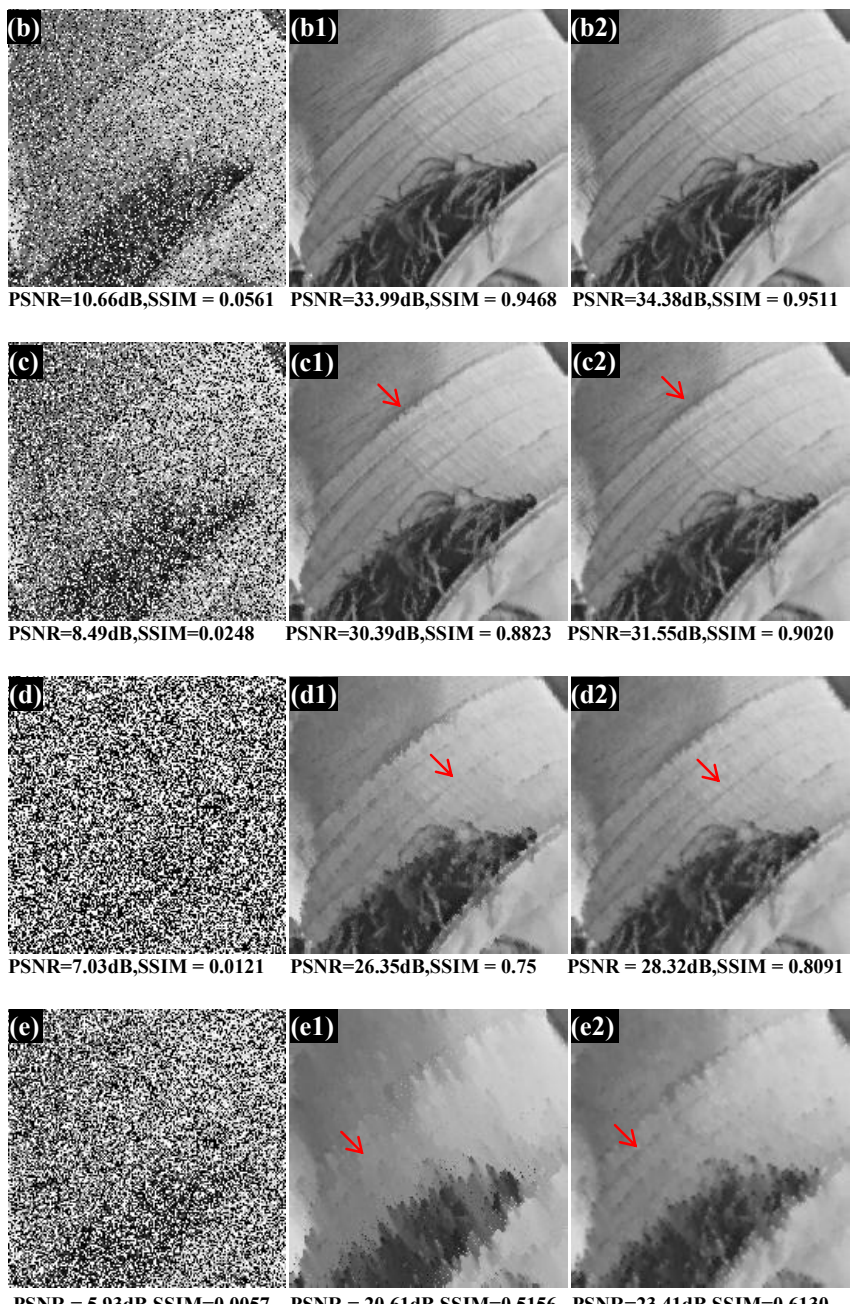

PSNR $=5.93 \mathrm{~dB}, \mathrm{SSIM}=0.0057 \quad \mathrm{PSNR}=20.61 \mathrm{~dB}, \mathrm{SSIM}=0.5156 \quad \mathrm{PSNR}=23.41 \mathrm{~dB}, \mathrm{SSIM}=0.6130$

Fig. 5. Restoration results for Lena image (30\%-90\% noise densities). (a) the original image; (b), (c), (d) and (e) are the corrupted images with $30 \%$, $50 \%$, $70 \%$ and $90 \%$ noise densities; (b1), (c1), (d1), (e1) are the median filter processed images of the noisy images in (b), (c), (d) and (e); (b2), (c2), (d2), (e2) are the average filter processed images of the noisy images in (b), (c), (d) and (e). 

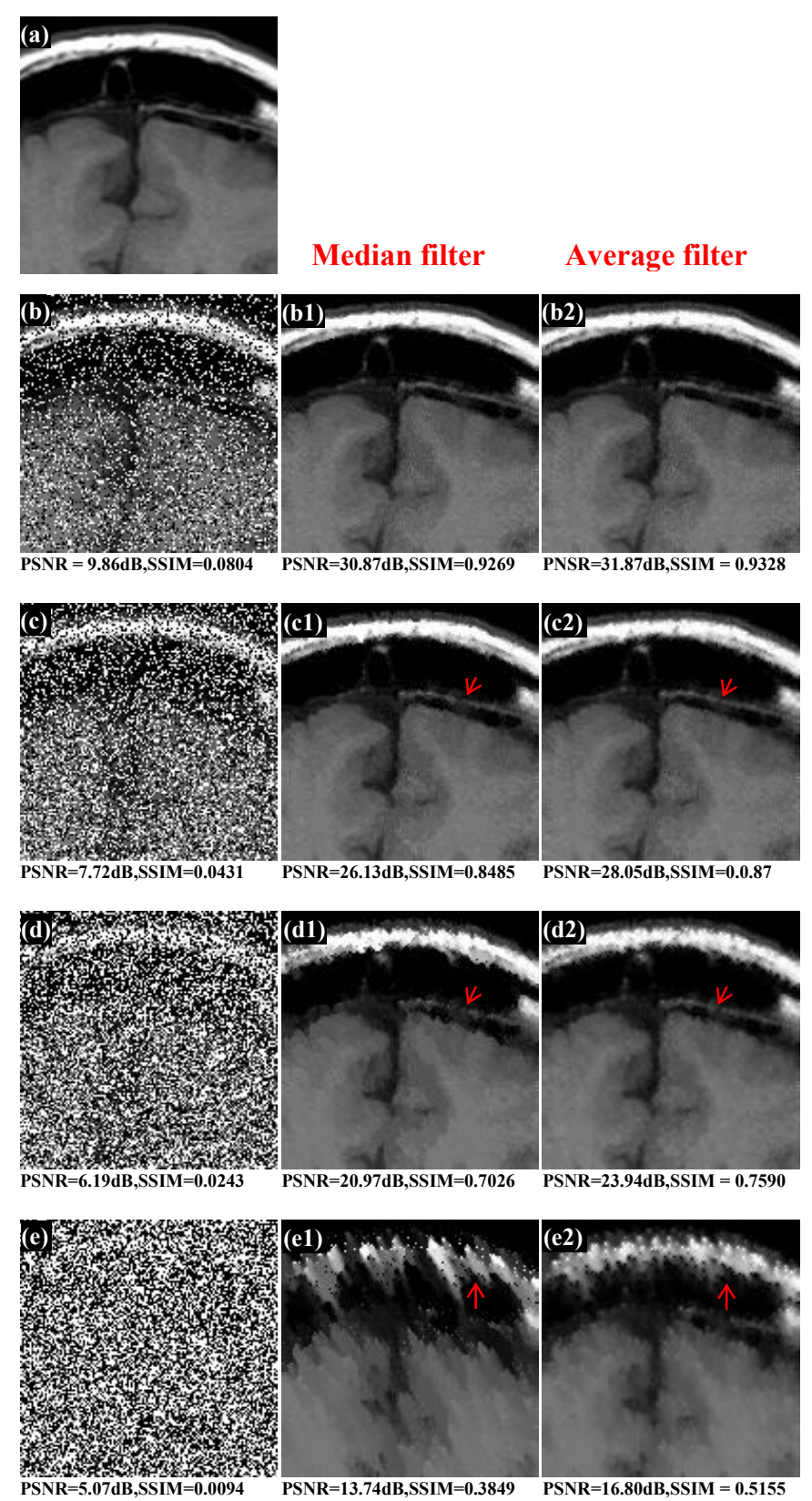

Fig. 6. Restoration results for MRI image (30\%-90\% noise densities). (a) the original image; (b), (c), (d) and (e) are the corrupted images with 30\%, 50\%, 70\% and $90 \%$ noise densities; (b1), (c1), (d1), (e1) are the median filter processed images of the noisy images in (b), (c), (d) and (e); (b2), (c2), (d2), (e2) are the average filter processed images of the noisy images in (b), (c), (d) and (e).

\section{CONCLUSION}

The exact distribution for the 2D neighboring intensities is always unknown in pixel estimation. Tough median based filters are widely used in suppression impulse noise, this paper provides some clue that Gaussian distribution based average filtering might be preferable to Laplacian distribution based median filtering in estimating missing data with unknown distribution. 
We can also see in Fig. 5 and Fig. 6 that the average filtering tends to produce artifacts in restoration. The reason might be that a simple non-discriminatively identical variance is used in (3). Some discriminative weighting strategies or more complicated models should be considered to give more effective estimations [16-17]. A thorough comparison with some advanced median-based filters also needs to be performed.

\section{REFERENCES}

[1] A. Bovik, Handbook of Image and Video Processing. New York: Academic, 2000.

[2] J. Astola and P. Kuosmaneen, Fundamentals of Nonlinear Digital Filtering. Boca Raton, FL: CRC, 1997.

[3] S. Zhang and M. A. Karim, “A new impulse detector for switching median filters,” IEEE Signal Process. Lett., vol. 9, no. 11, pp. 360-363, 2002.

[4] X.-M. Zhang and Y.-L. Xiong, "Impulse noise removal using directional difference based noise detector and adaptive weighted mean filter," IEEE Signal Process. Lett., vol. 16, no. 4, pp. 295-298, 2009.

[5] P. E. Ng and K. K. Ma, “A switching median filter with boundary discriminative noise detection for extremely corrupted images,” IEEE Trans. Image Process., vol. 15 , no. 6 , pp. 1506-1516, 2006.

[6] H. Hwang and R. A. Hadded, “Adaptive median filter: New algorithms and results," IEEE Trans. Image Process., vol. 4, no. 4, pp. 499-502, 1995.

[7] K. S. Srinivasan and D. Ebenezer, "A new fast and efficient decision based algorithm for removal of high density impulse noise,” IEEE Signal Process. Lett., vol. 14, no. 3, pp. 189-192, 2007.

[8] S. Esakkirajan, T. Veerakumar, Adabala N. Subramanyam, and C. H. Prem Chand, "Removal of high density salt and pepper noise through modified decision based unsymmetric Trimmed Median Filter”, IEEE Signal Process. Lett., vol. 18, no. 5, pp. 287-290, 2011.

[9] R. H. Chan, C.-W. Ho, and M. Nikolova,"Salt and pepper noise removal by median type noise detectors and detail preserving regularization,"IEEE Trans. Image Process., vol. 14, no. 10, pp. 1479-1485,2005.

[10] P. Sree, P. Kumar, R. Siddavatam, R. Verma, "Salt-and-pepper noise removal by adaptive median-based lifting filter using second-generation wavelets", Signal, Image and Video Processing, vol. 7, no. 1, pp. 111-118, 2013.

[11] G. R. Arce, “A general weighted median filter structure admitting negative weights,” IEEE Trans. Signal Process., vol. 46, pp. $3195-3205$, 1998.

[12] J. W. Harris, and H. Stocker, "Maximum Likelihood Method." (§21.10.4) in Handbook of Mathematics and Computational Science. New York: Springer-Verlag, p. 824, 1998.

[13] B. I. Justusson, "Median filtering: Statistical properties," in Two-Dimensional Digital Signal Processing It, Transforms and Median Filters, Huang, T. S., Ed. New York: Springer, 1981.

[14] Y. H. Lee and S. Tantaratana, "A simple formula for the variance of the median filter output with Laplacian input," IEEE Trans. Signal Process., vol. 39, pp. $984-986,1991$.

[15] Z. Wang, Alan C. Bovik, Hamid R. Sheikh and Eero P. Simoncelli "Image quality assessment: from error visibility to structural similarity" IEEE Trans. Image Processing, vol. 13, no. 4, pp. 600-612, 2004.

[16] D. Andrews and C. Mallows, "Scale mixtures of normal distributions," J. Royal Stat. Soc., vol. 36, pp. 99-102, 1974.

[17] M. J. Wainwright and E. P. Simoncelli, "Scale mixtures of Gaussians and the statistics of natural images," in Adv. NeuralInformation Processing Systems 
(NIPS*99), S. A. Solla, T. K. 